\title{
Clinical outcome after particle therapy for meningiomas of the skull base: toxicity and local control in patients treated with active rasterscanning
}

Rami A. El Shafie ${ }^{1,7^{*}+}$, Maja Czech ${ }^{1,7+}$, Kerstin A. Kessel ${ }^{4,5,6,1}$, Daniel Habermehl ${ }^{4,5,6,1}$, Dorothea Weber ${ }^{8}$, Stefan Rieken ${ }^{1,3,7}$, Nina Bougatf ${ }^{1,3,7}$, Oliver Jäkel ${ }^{2,3}$, Jürgen Debus ${ }^{1,3,9}$ and Stephanie E. Combs ${ }^{4,5,6,1}$

\begin{abstract}
Background: Meningiomas of the skull base account for $25-30 \%$ of all meningiomas. Due to the complex structure of the cranial base and its close proximity to critical structures, surgery is often associated with substantial morbidity. Treatment options include observation, aggressive surgical intervention, stereotactic or conventional radiotherapy.

In this analysis we evaluate the outcome of 110 patients with meningiomas of the skull base treated with particle therapy. It was performed within the framework of the "clinical research group heavy ion therapy" and supported by the German Research Council (DFG, KFO 214).

Methods: Between May 2010 and November 2014, 110 Patients with skull base meningioma were treated with particle radiotherapy at the Heidelberg lon Therapy Center (HIT). Primary localizations included the sphenoid wing $(n=42)$, petroclival region $(n=23)$, cavernous sinus $(n=4)$, sella $(n=10)$ and olfactory nerve $(n=4)$. Sixty meningiomas were benign ( $\mathrm{WHO}^{\circ}$ )); whereas 8 were high-risk (WHO ${ }^{\circ} \|(n=7)$ and $\left.{ }^{\circ} \| I(n=1)\right)$. In 42 cases histology was not examined, since no surgery was performed.

Proton ( $n=104)$ or carbon ion $(n=6)$ radiotherapy was applied at Heidelberg lon Therapy Center (HIT) using rasterscanning technique for active beam delivery. Fifty one patients (46.4\%) received radiotherapy due to tumor progression, 17 (15.5\%) after surgical resection and 42 (38.2\%) as primary treatment.

Results: Median follow-up in this analysis was 46,8 months (95\% Cl 39,9-53,7; Q1-Q3 34,3-61,7). Particle radiotherapy could be performed safely without toxicity-related interruptions. No grade IV or $V$ toxicities according to CTCAE v4.0 were observed. Particle RT offered excellent overall local control rates with 100\% progression-free survival (PFS) after 36 months and $96.6 \%$ after 60 months. Median PFS was not reached due to the small number of events. Histology significantly impacted PFS with superior PFS after 5 years for low-risk tumors $(96.6 \%$ vs. $75.0 \%$, $p=0,02$ ). Overall survival was $96.2 \%$ after 60 months and $92.0 \%$ after 72 months from therapy. Of six documented deaths, five were definitely not and the sixth probably not meningioma-related.

\footnotetext{
* Correspondence: rami.elshafie@med.uni-heidelberg.de

${ }^{\dagger}$ Equal contributors

${ }^{1}$ Department of Radiation Oncology, University Hospital of Heidelberg, Im Neuenheimer Feld 400, 69120 Heidelberg, Germany

${ }^{7}$ Heidelberg Institute for Radiation Oncology (HIRO), Im Neuenheimer Feld 400, 69120 Heidelberg, Germany

Full list of author information is available at the end of the article
} 
(Continued from previous page)

Conclusion: Particle radiotherapy is an excellent treatment option for patients with meningiomas of the skull base and can lead to long-term tumor control with minimal side effects. Other prospective studies with longer follow-up will be necessary to further confirm the role of particle radiotherapy in skull base meningioma.

Keywords: Proton therapy, Carbon ion therapy, Active raster-scanning, Skull base, Toxicity, Quality of life, Radiotherapy, Radiotolerance, Benign, Malignant

\section{Background}

Meningiomas account for approximately one third of all primary brain tumors and tumors of the central nervous system [1]. Most are benign, slow growing lesions originating from the arachnoidal cap cells, with the skull base being the most frequent localization [2]. Besides benign histology, a smaller number of meningiomas can be of atypical or anaplastic histology, characterized by aggressive growth patterns and a high rate of recurrence [3]. Many analyses focus on meningiomas of the skull base because of its intricate anatomy and the close proximity to vascular structures, cranial nerves and the brainstem; consequently treatment in those cases is challenging and treatment options are controversially discussed.

Surgical resection has for long been the treatment of choice but in the last decades advances in radiotherapy (RT) such as radiosurgery, fractionated stereotactic radiotherapy (FSRT), or intensity-modulated radiotherapy (IMRT) have made radiotherapy an important treatment alternative $[4,5]$. Due to the complex anatomy of the skull base, tumor adherence to bony structures and the close proximity to sensitive organs at risk (OAR), total resection is often not possible as it may cause to substantial morbidity. Consequently, as neurosurgical resection is subtotal in many cases, it cannot achieve high long-term local control and overall survival rates [6]. Additional radiotherapy can improve chances for longterm tumor control [7].

Meningiomas are often discovered incidentally or present with only mild symptoms and indolent growth patterns. In those cases, there is no urgent need for therapeutic intervention. Active surveillance can be a reasonable approach, focusing on precise high-resolution contrast-enhanced magnetic resonance imaging (MRI) and clinical examinations in regular intervals (e.g. every six to 12 months, provided an asymptomatic/stable clinical situation). However, if the tumor enlarges significantly during the course of neuroimaging or clinical symptoms develop or worsen, treatment becomes mandatory [6].

Particle beams, such as protons and heavier ion beams like carbon ions offer high precision when it comes to dose application to the tumor volume so that OAR can be very effectively spared $[8,9]$.

With its unique physical characteristics, including the inverted dose profile, high local dose deposition within the Bragg Peak and a steep falloff outside the treatment volume, particle radiation therapy leads to a greater dose conformity than photon RT [10]. Compared to protons, carbon ions additionally offer the advantage of higher biological efficiency with a relative biological effectiveness (RBE) ranging between 3 and 5, potentially leading to higher local control rates [11].

To date, particle therapy at Heidelberg Ion Therapy Center (HIT) has been integrated into the clinical environment at our institution for close to a decade and is constantly being validated for the treatment of skull base meningiomas. In this present study, we analyse our results for skull base meningiomas in 110 patients treated with particle therapy - protons as well as carbon ions with special focus on treatment outcome and toxicity.

\section{Methods}

\section{Patient characteristics and histology}

In the present analysis we included 110 consecutive patients with meningiomas of the skull base that had not previously received radiotherapy. All patients received particle therapy - either with proton beams or carbon ion beams - at the Heidelberg Ion Therapy Center (HIT). One hundred four patients received proton therapy, 6 patients received carbon ion radiotherapy. All patients were enrolled in a close follow-up program, consisting of neuroimaging as well as clinical-neurological assessments. According to WHO classification, 60 (54.5\%) meningiomas were categorized as benign (WHO grade I). In 8 patients (7.3\%) a high-risk histology was observed, including 7 (6.4\%) WHO grade II and $1(0.9 \%)$ WHO grade III. In a total of 42 patients (38.2\%) histology was unknown, since surgery was not performed. The diagnosis in these cases was based on clinical presentation in combination with imaging, consisting of MRI as well as computed tomography (CT), revealing the typical attributes of meningiomas. In some cases an additional FET or Ga68DOTATOC-PET examination was performed to support the diagnosis.

\section{Tumor location}

The exact locations of the meningiomas were ascertained by reviewing all images used for treatment planning. Often, tumors had ambiguous extent into structures of the skull base and extended into several 
regions. In those cases we focused on main tumor extension and tumor origin to create a common classification.Patient characteristics, histology and tumor locations are listed in Table 1.

Table 1 Patient characteristics

\begin{tabular}{|c|c|c|}
\hline Patient characteristics & $n=$ & Percent \\
\hline \multicolumn{3}{|l|}{ Gender } \\
\hline male & 22 & $20.0 \%$ \\
\hline female & 88 & $80.0 \%$ \\
\hline \multicolumn{3}{|l|}{ Age at radiotherapy } \\
\hline Mean (SD) & 53 & 11.7 \\
\hline Median (Q1-Q3) & 52 & $29-85$ \\
\hline Median (range) & 52 & $45-59$ \\
\hline \multicolumn{3}{|l|}{ Histology } \\
\hline WHO I & 60 & $54.5 \%$ \\
\hline WHO ॥ & 7 & $6.4 \%$ \\
\hline WHO III & 1 & $0.9 \%$ \\
\hline unknown & 42 & $38.2 \%$ \\
\hline \multicolumn{3}{|l|}{ Location } \\
\hline sphenoid wing & 42 & $38.2 \%$ \\
\hline petroclival & 23 & $20.9 \%$ \\
\hline sphenoorbital & 13 & $11.8 \%$ \\
\hline supra- and/or parasellar region & 10 & $9.1 \%$ \\
\hline sinus cavernosus & 4 & $3.6 \%$ \\
\hline olfactory & 4 & $3.6 \%$ \\
\hline tentorial fold & 3 & $2.7 \%$ \\
\hline petrosal & 5 & $4.5 \%$ \\
\hline cerebello-pontine angle & 3 & $2.7 \%$ \\
\hline foramen magnum & 3 & $2.7 \%$ \\
\hline \multicolumn{3}{|l|}{ Karnofsky performance score } \\
\hline$\geq 80 \%$ & 101 & $91.8 \%$ \\
\hline$<80 \%$ & 9 & $8.2 \%$ \\
\hline \multicolumn{3}{|l|}{ Previous surgery } \\
\hline partial resection & 62 & $56.4 \%$ \\
\hline no surgery & 32 & $29.1 \%$ \\
\hline unknown & 9 & $8.2 \%$ \\
\hline biopsy & 4 & $3.6 \%$ \\
\hline complete resection & 3 & $2.7 \%$ \\
\hline \multicolumn{3}{|l|}{ treatment setting } \\
\hline due to tumor progression & 51 & $46.4 \%$ \\
\hline definite & 42 & $38.2 \%$ \\
\hline adjuvant / additive & 17 & $15.5 \%$ \\
\hline \multicolumn{3}{|l|}{ Particle therapy } \\
\hline protons & 104 & $94.5 \%$ \\
\hline carbon ions & 6 & $5.5 \%$ \\
\hline
\end{tabular}

\section{Previous surgery}

In most patients, previous neurosurgical intervention had been performed: 69 patients $(62.7 \%)$ had at least one surgical intervention, which was either subtotal or a biopsy in 66 patients and complete in only 3 patients. Nine patients had an unknown course of surgery. In 29 patients (26.4\%) undergoing surgery, more than one intervention had been performed in the past and 8 patients (7.3\%) received three or more interventions. In a total of 51 cases (46.4\%) RT was performed due to tumor progression, 42 patients $(38.2 \%)$ received radiation therapy as a definite treatment with some patients undergoing a biopsy beforehand. In 17 patients (15.5\%) $\mathrm{RT}$ was performed after surgical resection.

\section{Pre-treatment imaging}

For immobilization purposes, an individual custom-made thermoplastic mask was used throughout the entire treatment program. Treatment planning was based on a highresolution CT scan (native and contrast-enhanced, $3 \mathrm{~mm}$ slice thickness). For target volume definition the treatment planning CT imaging data was in all cases matched to a contrast-enhanced MRI with a maximum slice thickness of $3 \mathrm{~mm}$, including a T1-weighted contrast-enhanced sequence to allow for a more precise estimate of tumor extension. In 52 cases (47.3\%) an additional FET- and/or DOTATOC-PET was performed to further facilitate target volume definition. Particle irradiation was delivered using active rasterscanning for both protons and carbon ions, applying one fraction a day, 6 days a week.

\section{Treatment planning for proton therapy}

Low-risk meningiomas (WHO grade I or unknown, $n=$ 102) were treated with proton therapy. Two patients with higher grade meningeomas (grade II $n=1$, grade III $n=1$ ) were also treated with proton therapy. Those patients had previously received radiotherapy for other cranial tumors (retinoblastoma in one case and a different meningioma in a non-overlapping area in the other) and the moderately hypofractionated approach of carbon ion radiotherapy (3 Gy(RBE) per fraction) combined with the higher biological effectiveness was deemed not ideal in those cases with previous dose to OAR. Proton therapy was thus preferred in those two cases. Target volume delineation for proton therapy was performed as follows: On the T1weighted sequence contrasted tumor formations were delineated as gross tumor volume (GTV). To define the clinical target volume (CTV) a safety margin of 1-2 mm (benign histology) or $5 \mathrm{~mm}$ (malignant histology) was added and adapted at the discretion of the treating physician including adjoining meningeal enhancement (dural tail) and areas of potential microscopic spread. An isotropic PTV margin of $3 \mathrm{~mm}$ was added in all cases to compensate for positioning and technical insecurities, as 
is standard procedure for intracranial irradiation at HIT. Details of resulting target volume sizes are illustrated in Table 2. Generally, coverage by the prescribed dose was optimized for CTV; focally reduced PTV coverage was tolerated to allow for OAR sparing in cases of necessity. Median cumulative dose for proton irradiation was $54 \mathrm{~Gy}(\mathrm{RBE})$ (range 50-60 Gy(RBE)) at a dose per fraction of $1,8(n=57)$ or $2(n=47) \mathrm{Gy}(\mathrm{RBE})$.

\section{Treatment planning for carbon ion therapy}

High-risk meningeomas (WHO grades II and III, $n=6$ ) were treated with a carbon ion boost after having received a median cumulative dose of $50 \mathrm{~Gy}$ (range 48,4-55,8 Gy) of photon irradiation. Target volume delineation and dose prescription were performed analogous to the MARCIE trial, a prospective trial being conducted at our institution for the treatment of atypical meningeomas [3]. For carbon ion irradiation contrast-enhanced areas on the T1-weighted MRI sequence were delineated as GTV with a $5 \mathrm{~mm}$ CTV margin that could be adapted at the discretion of the treating physician (e.g. to respect anatomic boundaries). An isotropic PTV margin of $3 \mathrm{~mm}$ was added as explained earlier. Details of resulting target volume sizes are illustrated in Table 2. Carbon ion dose prescribed in all cases was $18 \mathrm{~Gy}(\mathrm{RBE})$ at a dose per fraction of $3 \mathrm{~Gy}(\mathrm{RBE})$.

\section{Follow-up procedure}

Patients were followed up prospectively after completion of particle therapy as described previously [12]. Clinical examinations, including ophthalmological and neurological evaluations if needed and contrast-enhanced MR imaging were scheduled initially 6 weeks after RT. Thereafter, patients were followed up every 3 months in the first year and then every 6-12 months in the following years when no clinical or imaging-based signs of tumor progression occurred. Procedure for every followup visit would consist of a contrast-enhanced MRI as well as thorough clinical check-up. To complete or update follow-up information, we contacted patients lost to follow-up directly by phone or correspondence asking for clinical neurological assessments as well as current

Table 2 Target volume sizes

\begin{tabular}{|c|c|c|c|c|}
\hline & median (ml) & Q1-Q3 (ml) & mean $(\mathrm{ml})$ & std dev $(\mathrm{ml})$ \\
\hline \multicolumn{5}{|c|}{ Target volumes for proton irradiation } \\
\hline GTV & 22,6 & $12,5-35,1$ & 28,7 & 25,9 \\
\hline CTV & 31,5 & $19,2-49,9$ & 44,5 & 42,4 \\
\hline PTV & 50,8 & $32,7-76,3$ & 68,0 & 57,5 \\
\hline \multicolumn{5}{|c|}{ Target volumes for carbon ion boost irradiation } \\
\hline GTV & 54,6 & $38,4-59,8$ & 47,3 & 22,3 \\
\hline CTV & 46,9 & $42-77,8$ & 58,5 & 29,3 \\
\hline PTV & 65,7 & $59,6-112$ & 82,1 & 38,8 \\
\hline
\end{tabular}

medical imaging. Four patients could not be contacted and were lost to follow-up.

Symptoms and toxicities were documented in detail in the patient's medical record and subsequently entered into a prospective research database maintained at our institution for long-term systematic follow-up of radiooncological patients [12]. Symptoms were classified according to the Common Terminology Criteria for Adverse Events (CTCAE) v4.0 [13]. New or worsening symptoms were considered acute and treatment-related toxicities if they occurred within the first 6 months after radiotherapy and late toxicities if they occurred after that. Symptoms were followed up and outcome was judged clinically as either stable/improved or worsened. Toxicities of grades I and II according to CTCAE were classified low-grade. Any de novo symptoms grade III or higher were classified highgrade, as were any pre-existing symptoms worsening by at least two CTCAE grades except if directly attributable to tumor progression.

\section{Statistics}

For descriptive baseline analyses, continous variables are given as means (SD) and median (quartiles, range where appropriate) and categorical variables as absolute and relative frequencies. The median follow-up time was calculated using the reverse Kaplan-Meier method [14]. Overall survival (OS) and progression-free survival (PFS) were both determined using the actuarial method of Kaplan-Meier. OS was calculated from the date of the first diagnosis to last follow-up or death and separately from the beginning of radiotherapy to last follow-up or death. PFS was determined from the day of first RT to the date of occurrence of one of the following: last follow-up/tumor progression/death. Survival curves for prognostic factors were compared using a two-sided logrank test. Since this was a retrospective exploratory data analysis $p$-values are of descriptive nature. A descriptive p-value of $<0.05$ was considered to indicate statistical significance. Toxicity was classified according to CTCAE $\mathrm{v} 4.0$ and assessed descriptively, giving absolute and relative frequencies for each symptom. Improvement or worsening of a certain symptom was judged clinically on a case-by-case basis. Statistical analyses were performed with the software IBM SPSS Statistics Version 22 (New York, USA).

\section{Results}

\section{Local tumor control and survival}

The reverse Kaplan-Meier estimate for median followup was 46,8 months (95\% CI 39,9-53,7; Q1-Q3 34,3$61,7)$ for progression-free survival and 57,97 month (95\% CI 50,6-62,5; Q1-Q3 38,3-68,9) for overall survival. Progression-free survival rate for all patients treated with particle therapy was $100 \%$ after 36 months 
and $96.6 \%$ after 60 months. In total, four patients showed local progression. Median PFS was not reached due to the small number of events. Median time to progression was 55,6 months (Q1-Q3 45,2-65,1; range 40,0-67,3 months) (Fig. 1). Histology significantly impacted PFS with inferior PFS at 60 months $(75.0 \%$ for high-risk vs. $96.6 \%$ for low-risk histology, $p=0,02$ ) (Fig. 2) , although notably there were only eight patients and one event in the high-risk group, adding to the fact that histology at primary diagnosis was unknown for $38.2 \%$ of the patients and thus limiting the conclusions to be drawn on this data regarding the impact of histology.

Overall survival from the beginning of particle therapy was $96.2 \%$ after 60 months and $92.0 \%$ after 72 months. Median OS was not reached due the small number of events (Fig. 3). OS from the date of initial diagnosis was $98.1 \%$ after 10 years and $90.7 \%$ after 15 years (Fig. 4). In total, there were six deaths, for five of which the cause could reliably be determined. None of those deaths was meningioma-related. Two patients died of other oncologic illnesses (pancreatic $(n=1)$ and ovarian cancer $(n=1))$. Two patients died of substantial cardiovascular co-morbidities and one patient died of a pre-existing advanced-stage normal pressure hydrocephalus. The latter three patients had already been severely reduced in general performance prior to therapy. Four deaths occurred within the first 3 years of radiotherapy. Five deaths occurred in the low-risk group and one in the highrisk group. None of the patients who died had progressed at the last recorded follow-up.

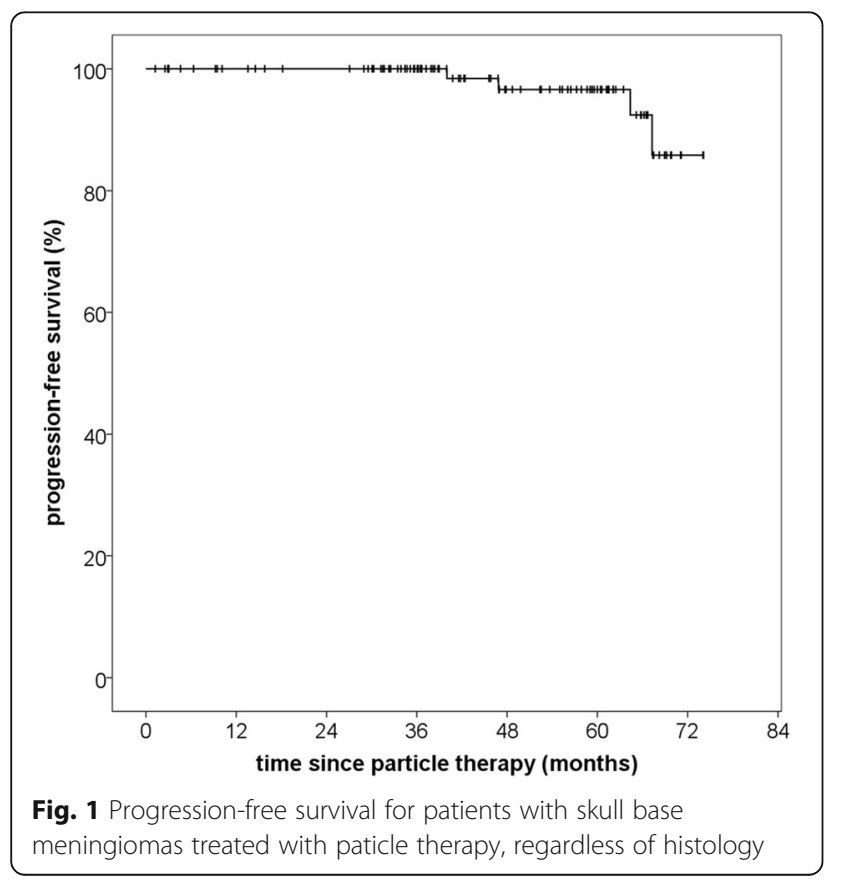

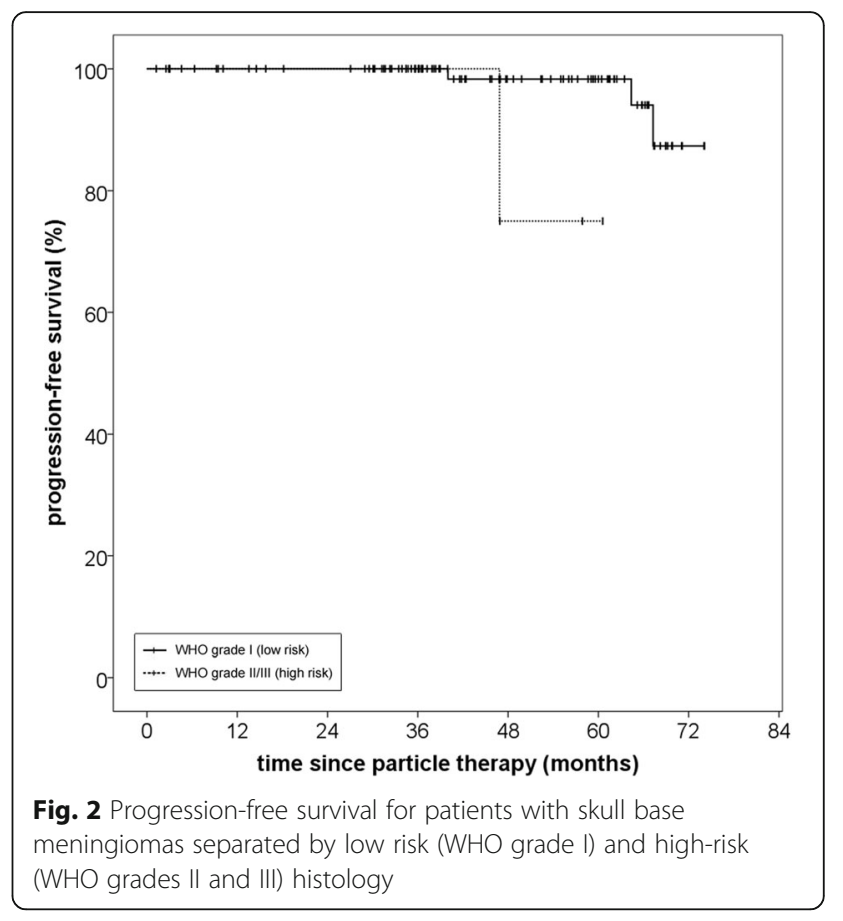

\section{Progressing patients}

The four patients progressing after particle RT were characterized as follows: The first patient was treated for a meningioma of the intrasellar region in a definite setting with $32 \times 1,8 \mathrm{~Gy}(\mathrm{RBE})$ of proton irradiation without prior surgery or biopsy. She progressed locally 39 months thereafter and underwent partial resection, showing a grade II meningioma, and afterwards received additive

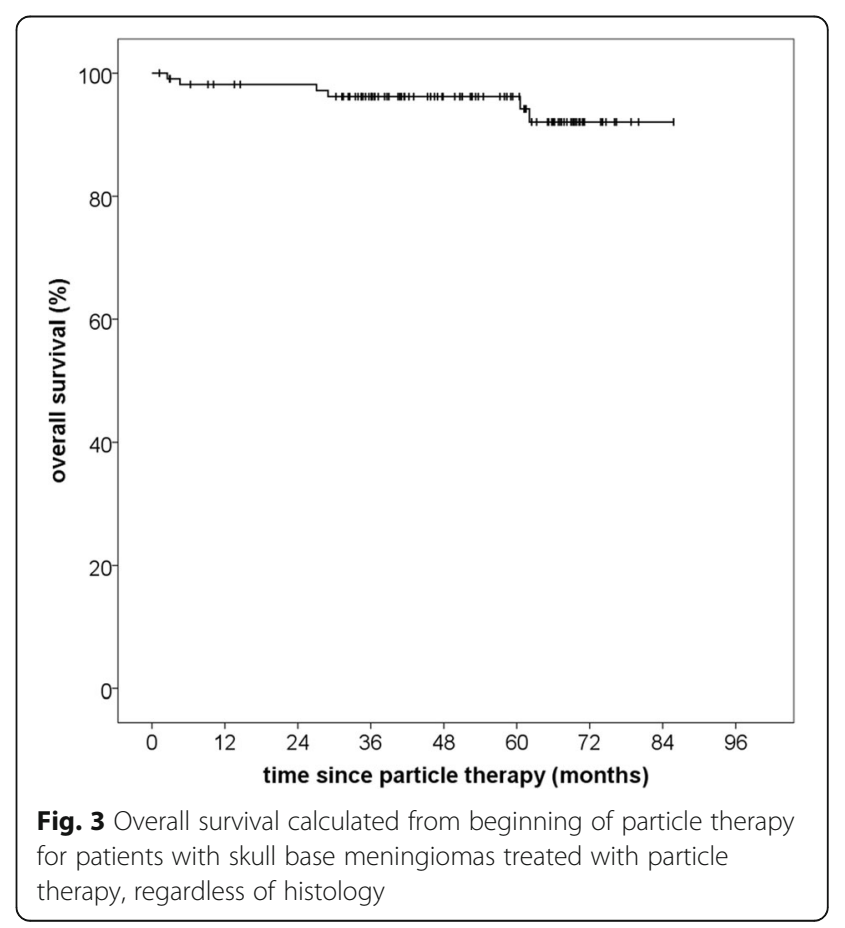




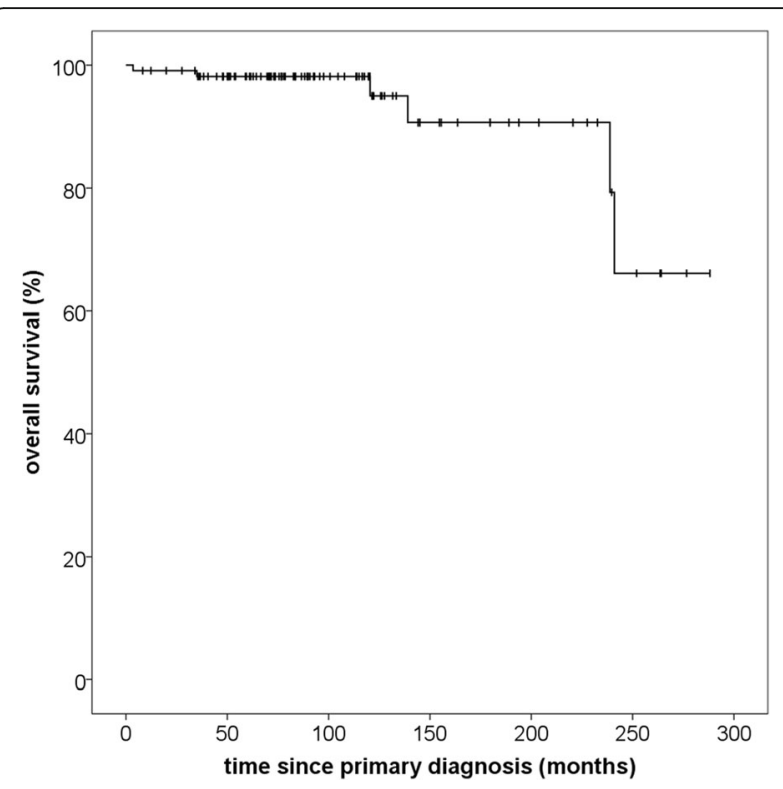

Fig. 4 Overall survival calculated from primary diagnosis for patients with skull base meningiomas treated with particle therapy, regardless of histology

re-irradiation with $15 \times 3 \mathrm{~Gy}(\mathrm{RBE})$ carbon ion therapy. The second patient received $25 \times 2$ Gy photon IMRT and a carbon ion boost of $18 \mathrm{~Gy}(\mathrm{RBE})$ (cumulative dose $68 \mathrm{~Gy}(\mathrm{RBE}))$ after multiple partial resections of a petrosal WHO grade II meningioma. Local progression occurred after 47 months and was treated with $15 \times$ $3 \mathrm{~Gy}(\mathrm{RBE})$ carbon ions as re-irradiation. He showed no further tumor progression until last follow-up 17 months thereafter. The third patient was treated with $28 \times$ $2 \mathrm{~Gy}(\mathrm{RBE})$ of proton irradiation for a meningioma of unknown histology located in the left sphenoid wing and parasellar region. Local progression occurred after 63 months and the patient was referred to neurosurgical resection. The fourth patient received $27 \times 2 \mathrm{~Gy}(\mathrm{RBE})$ of proton irradiation for a partially resected WHO grade I meningioma of the sphenoorbital region. Local progression occurred after 66 months and the patient was referred to neurosurgical resection.

\section{Treatment-related toxicity}

Treatment was overall well tolerated. All patients completed treatment successfully and no interruptions for toxicity-associated reasons were necessary. No treatmentrelated grade IV or V toxicities according to CTCAE v4.0 were observed. Acute treatment-related toxicity was mild and mostly resolved within the first 6 months after therapy completion. Among the most common symptoms were focal alopecia (63.6\%, $n=70)$, moderate fatigue $(47.3 \%, n=$ $52)$, focal skin irritation $(40.0 \%, n=44)$ and headaches (22. $7 \%, n=25)$. There were two cases of acute grade III toxicity: One case of severe ulcerating mucositis requiring hospitalization and one case of prolonged nausea due to intracranial pressure requiring the administration of corticosteroids. Both cases were controllable by supportive medication. The most common symptoms among late toxicity were fatigue and headaches (both $9.1 \%, n=10$ ). No more than 10 patients reported any one symptom as late toxicity. Overall, four cases of late CTCAE grade III toxicity were reported: One patient with a parasellar/intrasellar meningeoma developed massively progressive fatigue for which the reason proved to be radiogenic hypopituitarism. Symptoms were controlled after endocrinologic follow-up and adequate medication. Three patients developed radionecrosis, two of them symptomatic (headaches, dizziness). Delivered doses were $30 \times 1,8 \mathrm{~Gy}(\mathrm{RBE})$ protons $(\mathrm{n}=1$, interval $=9$ months), $27 \times 2 \mathrm{~Gy}(\mathrm{RBE})$ protons $(\mathrm{n}=1$, inter$\mathrm{val}=36$ months) and $5 \times 3 \mathrm{~Gy}(\mathrm{RBE})$ carbon ion boost after 50 Gy photon IMRT ( $n=1$, interval $=7$ months). To treat radionecrosis, two patients received high-dose corticosteroids and one patient received Bevacizumab under which clinical and radiologic response could be observed. An overview of acute and late treatment-related toxicity including respective CTCAE gradings is presented in Table 3.

\section{Symptom response to treatment}

The majority of patients presented with multiple symptoms pre-existing radiation therapy which included motoric and sensory impairment, as well as partial trigeminal and facial nerve paralysis, hearing impairment, headache and dizziness. The most common symptom prior to radiotherapy proved to be visual impairment, mostly double vision in 45 patients. Moreover, pre-existing double vision and headache prior to radiotherapy showed the most sizeable improvement, with symptom improvement or stabilization in $34.5 \%(n=38)$ for vision impairment and $41.8 \%(n=46)$ for headaches. These improvements of pretherapeutic symptoms were achieved within a year after irradiation. No more than $8.2 \%(n=9)$ of all patients reported a worsening of any given symptom. The most common among worsening symptoms after RT were headaches $(8.2 \%, n=9)$ and fatigue $(7.3 \%, n=8)$. An overview of predominant symptoms prior to particle therapy, their grading according to CTCAE $v 4.0$ and their relative development during follow-up is presented in Table 4.

\section{Discussion}

The present manuscript evaluates the efficacy and toxicity profile of particle therapy for the treatment of 110 consecutive patients over a a period of 5 years, treated at a single institution. Histology was predominantly benign (WHO grade I) and mainly proton therapy was used, although a combination of photon IMRT and a carbon ion boost was used for a total of six patients with highergrade histology. None of the treated meningiomas had 
Table 3 Acute and late treatment-related toxicity

\begin{tabular}{|c|c|c|c|c|c|c|c|c|}
\hline \multirow{3}{*}{ Side effect } & \multicolumn{4}{|c|}{ Acute treatment-related toxicity } & \multicolumn{4}{|c|}{ Late treatment-related toxicity } \\
\hline & \multicolumn{2}{|c|}{ low grade (CTCAE I-II) } & \multicolumn{2}{|c|}{ high grade (CTCAE III or higher) } & \multicolumn{2}{|c|}{ low grade (CTCAE I-II) } & \multicolumn{2}{|c|}{ high grade (CTCAE III or higher) } \\
\hline & $n=$ & $\%$ & $n=$ & $\%$ & $n=$ & $\%$ & $n=$ & $\%$ \\
\hline focal alopecia & 70 & $63.6 \%$ & 0 & $0.0 \%$ & 1 & $0.9 \%$ & 0 & $0.0 \%$ \\
\hline fatigue & 52 & $47.3 \%$ & 0 & $0.0 \%$ & 10 & $9.1 \%$ & 1 & $0.9 \%$ \\
\hline skin irritation & 44 & $40.0 \%$ & 0 & $0.0 \%$ & 0 & $0.0 \%$ & 0 & $0.0 \%$ \\
\hline headache & 25 & $22.7 \%$ & 0 & $0.0 \%$ & 10 & $9.1 \%$ & 0 & $0.0 \%$ \\
\hline nausea & 23 & $20.9 \%$ & 1 & $0.9 \%$ & 0 & $0.0 \%$ & 0 & $0.0 \%$ \\
\hline facial pain & 12 & $10.9 \%$ & 0 & $0.0 \%$ & 2 & $1.8 \%$ & 0 & $0.0 \%$ \\
\hline dysgeusia & 8 & $7.3 \%$ & 0 & $0.0 \%$ & 4 & $3.6 \%$ & 0 & $0.0 \%$ \\
\hline lymphedema & 7 & $6.4 \%$ & 0 & $0.0 \%$ & 3 & $2.7 \%$ & 0 & $0.0 \%$ \\
\hline xerostomia & 5 & $4.5 \%$ & 0 & $0.0 \%$ & 4 & $3.6 \%$ & 0 & $0.0 \%$ \\
\hline mucositis & 1 & $0.9 \%$ & 1 & $0.9 \%$ & 1 & $0.9 \%$ & 0 & $0.0 \%$ \\
\hline radionecrosis & 0 & $0.0 \%$ & 0 & $0.0 \%$ & 0 & $0.0 \%$ & 3 & $2.7 \%$ \\
\hline
\end{tabular}

previously been irradiated. Excellent overall local control at the cost of very light toxicity was achieved with $100 \%$ PFS after three and 96.6\% PFS after 5 years and histology appearing to significantly influence PFS.

The treatment of skull base meningiomas is a complex clinical situation that requires careful interdisciplinary evaluation. Due to the intricate anatomy of the skull base and the distinct subset of symptoms and toxicities caused by tumors there located, it has been discussed that skull base meningiomas should be regarded as a separate entity regarding outcome and treatment-associated toxicity [4].

Over the years, radiation therapy - and particularly high-precision techniques such as FSRT or IMRT - has evolved to become a central pillar in the multimodal treatment of meningiomas. Several groups have shown high efficacy with minimal toxicity $[4,6,15]$ : One of the largest collectives of skull base meningiomas treated with photon IMRT or FSRT and with a median followup of 107 months has been described at our institution, showing a local control rate of $95 \%$ at 5 years and $88 \%$ at 10 years [4]. Histology (WHO grade I vs. grades II and III) proved to be an important prognostic factor, significantly impacting PFS. These data have been confirmed by several similar studies performed at other institutions: Kaul et al. have described PFS to be $93.8 \%$ after 5 years for 318 patients with benign meningeomas treated with FSRT [16]. In a separate series focusing exclusively on skull base meningiomas PFS was similar for low-risk histology and $41.8 \%$ after 5 years for high-risk histologies [17]. Minniti et al. found a PFS rate of $96 \%$ at

Table 4 Predominant symptoms prior to particle radiotherapy and their relative improvement development during follow-up

\begin{tabular}{|c|c|c|c|c|c|c|c|c|c|c|c|c|}
\hline \multirow{3}{*}{$\begin{array}{l}\text { Predominant clinical } \\
\text { symptomps }\end{array}$} & \multicolumn{4}{|c|}{ Symptoms before particle irradiation } & \multicolumn{4}{|c|}{ Symptoms at last follow-up } & \multicolumn{4}{|c|}{ Clinical outcome } \\
\hline & \multicolumn{2}{|c|}{$\begin{array}{l}\text { low grade } \\
\text { (CTCAE I-II) }\end{array}$} & \multicolumn{2}{|c|}{$\begin{array}{l}\text { high grade } \\
\text { (CTCAE III or higher) }\end{array}$} & \multicolumn{2}{|c|}{$\begin{array}{l}\text { low grade } \\
\text { (CTCAE I-II) }\end{array}$} & \multicolumn{2}{|c|}{$\begin{array}{l}\text { high grade } \\
\text { (CTCAE III or higher) }\end{array}$} & \multicolumn{2}{|c|}{ stable or improvement } & \multicolumn{2}{|c|}{ worsening } \\
\hline & $n=$ & $\%$ & $n=$ & $\%$ & $n=$ & $\%$ & $n=$ & $\%$ & $n=$ & $\%$ & $n=$ & $\%$ \\
\hline visual impairment & 35 & $31.8 \%$ & 10 & $9.1 \%$ & 28 & $25.5 \%$ & 11 & $10.0 \%$ & 38 & $34.5 \%$ & 6 & $5.5 \%$ \\
\hline headaches & 35 & $31.8 \%$ & 0 & $0.0 \%$ & 27 & $24.5 \%$ & 1 & $0.9 \%$ & 46 & $41.8 \%$ & 9 & $8.12 \%$ \\
\hline hearing impairment & 25 & $22.7 \%$ & 3 & $2.7 \%$ & 25 & $22.7 \%$ & 2 & $1.8 \%$ & 33 & $30.0 \%$ & 3 & $2.7 \%$ \\
\hline motoric impairment & 19 & $17.3 \%$ & 1 & $0.9 \%$ & 15 & $13.6 \%$ & 2 & $1.8 \%$ & 20 & $18.2 \%$ & 1 & $0.9 \%$ \\
\hline sensory impairment & 14 & $12.7 \%$ & 0 & $0.0 \%$ & 12 & $10.9 \%$ & 0 & $0.0 \%$ & 16 & $14.5 \%$ & 2 & $1.8 \%$ \\
\hline dizziness & 13 & $11.8 \%$ & 0 & $0.0 \%$ & 9 & $8.2 \%$ & 0 & $0.0 \%$ & 21 & $19.1 \%$ & 2 & $1.8 \%$ \\
\hline facial pain & 13 & $11.8 \%$ & 0 & $0.0 \%$ & 9 & $8.2 \%$ & 0 & $0.0 \%$ & 21 & $19.1 \%$ & 2 & $1.8 \%$ \\
\hline cognitive impairment & 11 & $10.0 \%$ & 1 & $0.9 \%$ & 14 & $12.7 \%$ & 2 & $1.8 \%$ & 16 & $14.5 \%$ & 4 & $3.6 \%$ \\
\hline seizures & 9 & $8.2 \%$ & 1 & $0.9 \%$ & 2 & $1.8 \%$ & 1 & $0.9 \%$ & 9 & $8.2 \%$ & 1 & $0.9 \%$ \\
\hline fatigue & 6 & $5.5 \%$ & 0 & $0.0 \%$ & 15 & $13.6 \%$ & 1 & $0.9 \%$ & 42 & $38.2 \%$ & 8 & $7.3 \%$ \\
\hline nausea & 4 & $3.6 \%$ & 0 & $0.0 \%$ & 9 & $8,2 \%$ & 0 & $0,0 \%$ & 22 & $20.0 \%$ & 0 & $0.0 \%$ \\
\hline mucositis & 0 & $0.0 \%$ & 0 & $0.0 \%$ & 1 & $0,9 \%$ & 1 & $0,9 \%$ & 2 & $1.8 \%$ & 0 & $0.0 \%$ \\
\hline
\end{tabular}


3 years and $93 \%$ at 5 years in a series of 52 patients with large skull base meningiomas treated with FSRT [18]. Kessel et al. comprehensively reviewed recent literature on the subject and published another large series of 260 patients treated with FSRT or IMRT and including 16\% high-risk histologies. They found a PFS rate after 5 years of $87.1 \%$ and $54.9 \%$ for low-risk and high-risk histologies respectively. Futhermore, patient-reported outcome showed very mild toxicity with no more than $3.0 \%$ of patients experiencing worsened or new symptoms $\geq 3$ during RT and the first 6 months thereafter [15].Our results have shown that proton therapy can achieve similarly excellent local control, though continuous long-term follow-up is warranted. Reported data on toxicity and symptom response to treatment is very similar to the results achieved in the current analysis with only mild acute toxicity, the majority of patients showing either stable or improved symptoms during long-term follow-up.

One of the main rationales for the use of particle therapy lies in its higher dose conformity, potentially allowing for better OAR sparing and the reduction of side effects [19-21]. The energy-deposition of accelerated photons occurs continuously over a comparably wide range of penetration depths through tissue [22]. The improved dose distribution of particle therapy is achieved by exploiting the physical characteristics of particle irradiation where the maximum dose deposition occurs within the sharply defined Bragg peak [9]. By varying the particle energy, the position of the Bragg peak can be altered. Particle therapy has been shown to be superior to photon-based techiques in terms of sparing OAR and in terms of target dose homogeneity/conformity with carbon ions showing slightly superior dose distributions compared to protons [23, 24]. Arvold et al. observed a significant dose reduction to neurocognitive, visual and auditory organs achieved by proton irradiation as compared to photon RT. Furthermore, they found protons to reduce the risk of developing a radiologically-induced or associated secondary malignancy by half [1]. Other publications showed a sizeable improvement of pre-exstisting clinical symptoms in up to $47 \%$ of patients treated with proton radiotherapy for meningiomas, to which our results compare favorably [10,25]. We could observe a clear tendency towards improvement that was most prominent in patients suffering from visual impairment, mostly diplopia. $34.5 \%$ of all patients showed stabilization or improvement regarding eye-related symptoms and $41.8 \%$ regarding headaches, corresponding to $77.8 \%$ of the patients reporting pre-therapeutical eye-related symptoms and $94.3 \%$ of the patients reporting headaches respectively.

In recent years the body of literature on treating meningiomas with proton therapy has steadily grown and to date there are several publications describing adequatesized collectives with a median follow-up of 32 to
84 months: Vlachogiannis et al. recently published a retrospective analysis on 170 patients with grade I meningiomas, 155 of which were located at the skull base, who received hypofractionated proton therapy over a period of 13 years. Median follow-up was 84 months and authors reported PFS rates of $93 \%$ and $85 \%$ at five and 10 years respectively. Main differences in comparison to the current work were the use of passive scattering and the hypofractionated dose regime of 3-8 fractions at 5 or $6 \mathrm{~Gy}(\mathrm{RBE})$ per fraction, translating approximately to an EQD2 (equivalent dose in 2-Gyfractions) of $43 \mathrm{~Gy}$.

Halasz et al. were the first to describe a radiosurgical approach for proton therapy in meningiomas [10]. They analysed a group of 50 patients treated with proton stereotactic radiosurgery at a dose of 13 Gy prescribed to the $90 \%$ isodose, achieving a three-year acturial tumor control rate of $94 \%$ and toxicity rates similar to those described above. The regarded collective included only small tumor volumes and low-risk histologies. The data suggest that a hypofractionated or even radiosurgical approach, as has been extensively evaluated for photon therapy, might be a feasible and well-tolerated approach for proton therapy as well and achieve satisfactory results [26].

A recent retrospective study by Murray et al. described the outcome of 96 meningioma patients treated with pencil beam scanning proton therapy at Paul Scherrer Institute in Switzerland over a 10 year period [27]. 63.5\% were low-risk and and $36.5 \%$ high risk meningiomas. The authors reported an estimated 5-year local control (5y-LC) rate of $95.7 \%$ for the low-risk group and $68 \%$ for the high-risk group, showing consistency with the previously discussed literature and the results of our current work. Five-year grade $\geq 3$ toxicity-free survival was $89.1 \%$. The authors reported on identifying several prognostic factors for local failure beside histology $(p<0,001)$, One such factor was the timing of particle therapy (initial vs. for recurrence or progressive disease) with patients treated initially showing favorable outcome; furthermore tumors of the skull base showed favorable outcome vs. non-skull base $(p=0,14)$, as did female patients vs. males $(p=0,32)$. However, none of those factors was tested in multivariate analysis, thus their predictive value should be interpreted with care.

DiBiase and colleagues revealed the size of the GTV to be a significant prognostic factor, since in their described collective of 162 patients treated with Gamma Knife SRS, patients with smaller tumor volumes had longer survival rates with a 5 -year overall survival of $100 \%$ compared to $59.7 \%$ for larger lesions [28].

A small prospective randomized series by Sanford et al. has tested the effect of dose escalation using a combination of photon and proton therapy for the treatment 
of low-risk meningiomas with a median follow-up of 17,1 years [29]. While overall local control of $98 \%$ at 10 years and $90 \%$ at 15 years was excellent, no significant benefit could be shown for the use of $63 \mathrm{~Gy}(\mathrm{RBE})$ over 55,8 Gy(RBE). However, dose escalation might be beneficial for the treatment of high-risk meningioma patients who show less favorable outcomes with established dose regimens. 5y-LC rate for high-risk meningiomas was $75 \%$ in our analysis and though patient number was small, results are comparable to figures reported in recent literature of $50-81 \%$ for IMRT or proton therapy, depending on WHO grade [27, 30, 31].

Adeberg et al. could within the high-risk collective identify the WHO grade as prognostic factor for PFS with higher grade yielding inferior PFS $(p=0,017)$ [30]. Notably, the results of McDonald et al. support the rationale of dose escalation for high-risk meningiomas, achieving $5 y-\mathrm{LC}$ rates of $87.5 \%$ for a radiation dose $>60 \mathrm{~Gy}(\mathrm{RBE})$ compared to $50 \%$ for $\leq 60 \mathrm{~Gy}(\mathrm{RBE})$ of proton $\mathrm{RT}$ ( $p=$ $0,038)$ [31]. Regarding dose escalation in a highly radiosensitive region such as the skull-base, the use of heavier ions such as carbon ions with their potentially superior dose distribution and biological advantages attributed to the increased relative biological effiectiveness (RBE) could prove beneficial and may lead to higher local tumor control rates $[9,32]$. In a small prospective phase I/II trial conducted at our institution in 2010 on the administration of a carbon ion boost after photon radiotherapy for $10 \mathrm{pa}$ tients with high-risk meningiomas, we achieved promising results with 5- and 7-year local control rates of $86 \%$ and $72 \%$ [33]. Median cumulative dose in this analysis was $68 \mathrm{~Gy}(\mathrm{RBE})$ and the series included two previously irradiated tumors. Building upon those results, we have initiated the MARCIE trial, a prospective phase II trial evaluating PFS, OS and toxicity for the postoperative bimodal irradiation of atypical meningiomas Simpson grade 4 or 5 [3]. The trial is currently recruiting and the dose regimen of 50 Gy photon RT combined with $6 \times 3$ Gy carbon ion boost that we applied to the high-risk patients in this analysis is analogous to the concept employed in the MARCIE trial. Though patient number was small for high-risk meningiomas in our current analysis, results are in agreement with previously published data for this dose regimen [33].

Certainly a potential benefit of particle therapy over photon radiation techniques has to be verified clinically and prospective trials are warranted. Several treatment planning studies showed superiority for protons, particularly for larger target volumes: For example, Phillips et al. on reviewing different radiosurgical methods found that particle RT results in supervior dose distributions than photon-based linear accelarator (linac) methods for target volumes $>25 \mathrm{ccm}$, though for smaller volumes results are comparable while linac methods might offer higher flexibility [34, 35]. Smith et al. supported these findings, comparing linac-based photon RT to Gamma Knife SRS and proton $\mathrm{RT}$ and calculating normal tissue complication probability indices (NTCP) based on the dose conformity of the resulting treatment plans and using a logistic model based on the tolerance data by Rubin et al. and Emami et al. [36]. While photon SRS techniques proved superior for small spherical targets, protons had the lowest NTCP for large $(>15 \mathrm{ccm})$ and peripheral target volumes $(13,5$ for protons vs. 17,0-33,5 for linac) [37].

To date, our analysis represents the largest group of patients with skull base meningiomas treated with particle therapy, including both protons and carbon ions, in a single institution. Limitations of this analysis include the relatively short follow-up period, its retrospective character and the small number of both high-risk histologies and patients treated with carbon ions, limiting the possibility to perform meaningful subgroup analyses. The median follow-up in this series at 46,8 months - though substantial - is still relatively short when compared to other studies available, especially in the field of precision photon RT. In the light of the benign nature of low-grade meningiomas and predicted long-term tumor-control and overall survival continuous follow-up is warranted. Regarding the different physical and biological characteristics of particle therapy, potential long-term effects are of special interest. Currently particle therapy patients at our institution are included in a close-knit and rigorous follow-up regimen and potential late side effects are documented in a prospective database with dedicated institutional funding for long-term evaluation [12].

To conclusively demonstrate the clinical benefits of particle therapy there is currently a lack of a prospective comparison to advanced photons. Prospective clinical trials have since been initiated at several institutions to further establish the role of particle therapy for the treatment of certain subgroups of intracranial menigiomas.

\section{Conclusion}

In conclusion, particle therapy offers an excellent treatment option for patients with meningiomas of the skull base with long-term tumor control-rates and low toxicity. Compared to outcome reported on the treatment of skull base meningiomas in the literature, the results of our recent study compare favorably. Altough for this entity with its favorable outcome still longer follow-up is warranted, our results are in accordance with previous series of skull base meningiomas treated with particle therapy. Nonetheless, prospective studies with longer follow-up will be necessary to further confirm the role of particle radiotherapy in meningiomas of the skull base. Due to the excellent results with advanced photons an improvement in oncologic outcome by particle therapy in benign meningiomas will be difficult to demonstrate. 


\section{Abbreviations}

CTCAE: Common terminology criteria for adverse events; CTV: Clinical target volume; FSRT: Fractionated stereotactic radiotherapy; GTV: Gross tumor volume; IMRT: Intensity-modulated radiotherapy; MRI: Magnetic resonance imaging; OAR: Organ at risk; OS: Overall survival; PFS: Progression-free survival; PTV: Planning target volume; RBE: Relative biological effectiveness; RT: Radiotherapy; SRS: Stereotactic radiosurgery; WHO: World health organization

\section{Acknowledgements}

This work was presented at the German Society of Radiation Oncology (DEGRO) Annual Meeting in 2014.

\section{Funding}

This work was performed within the framework of the "clinical research group heavy ion therapy" and supported by the German Research Council (DFG, KFO 214).

\section{Availability of data and materials}

The datasets used and/or analysed during the current study are available from the corresponding author on reasonable request.

\section{Authors' contributions}

S.E. Combs, O. Jäkel and J. Debus developed and planned this analysis as part of the work of the Klinische Forschergruppe Schwerionentherapie (KFO 214). K. Kessel, M. Czech and N. Bougatf were responsible for data collection and data management. R.A. El Shafie, M. Czech, D. Habermehl and S. Rieken performed data analysis, endpoint evaluation and collaborated on writing the manuscript. D. Weber is the study biostatistician and reviewed all data and statistical analyses. All authors read and approved the final manuscript.

\section{Ethics approval and consent to participate}

The Heidelberg Ethics Committee approved this study on the 9th of July 2013 (S-207/2013)

\section{Consent for publication}

Not applicable.

\section{Competing interests}

The authors declare that they have no competing interests.

\section{Publisher's Note}

Springer Nature remains neutral with regard to jurisdictional claims in published maps and institutional affiliations.

\section{Author details}

'Department of Radiation Oncology, University Hospital of Heidelberg, Im Neuenheimer Feld 400, 69120 Heidelberg, Germany. ${ }^{2}$ Deutsches Krebsforschungszentrum (dkfz), Abteilung Medizinphysik, Im Neuenheimer Feld 270, 69120 Heidelberg, Germany. ${ }^{3}$ Heidelberg Ion Therapy Center (HIT), Im Neuenheimer Feld 470, 69120 Heidelberg, Germany. ${ }^{4}$ Department of Radiation Oncology, Klinikum rechts der Isar, Technische Universität München (TUM), Ismaninger Straße 22, 81675 Munich, Germany. ${ }^{5}$ Helmholtz Zentrum München, Department of Radiation Sciences (DRS), Institute of Innovative Radiotherapy (iRT), Ingolstädter Landstraße 1, Munich, Germany. ${ }^{6}$ Deutsches Konsortium für Translationale Krebsforschung (DKTK), Partner Site Munich, Munich, Germany. ${ }^{7}$ Heidelberg Institute for Radiation Oncology (HIRO), Im Neuenheimer Feld 400, 69120 Heidelberg, Germany. ${ }^{8}$ Institute for Medical Biometry and Informatics (IMBI), Heidelberg University Hospital, Im Neuenheimer Feld 130.3, 69120 Heidelberg, Germany. ${ }^{9}$ Clinical Cooperation Unit Radiation Oncology (E050), German Cancer Research Center (dkfz), Im Neuenheimer Feld 280, 69120 Heidelberg, Germany.

Received: 28 August 2017 Accepted: 16 March 2018

Published online: 27 March 2018

\section{References}

1. Arvold ND, Niemierko A, Broussard GP, Adams J, Fullerton B, Loeffler JS, et al. Projected second tumor risk and dose to Neurocognitive structures after proton versus photon radiotherapy for benign Meningioma. Int J Radiat Oncol. 2012;83:e495-500. https://doi.org/10.1016/j.jjrobp.2011.10.056.
2. Boskos C, Feuvret L, Noel G, Habrand J-L, Pommier P, Alapetite C, et al. Combined proton and photon conformal radiotherapy for intracranial atypical and malignant Meningioma. Int J Radiat Oncol. 2009;75:399-406. https://doi.org/10.1016/j.jprobp.2008.10.053.

3. Combs SE, Edler L, Burkholder I, Rieken S, Habermehl D, Jäkel O, et al. Treatment of patients with atypical meningiomas Simpson grade 4 and 5 with a carbon ion boost in combination with postoperative photon radiotherapy: the MARCIE trial. BMC Cancer. 2010;10:615. http://www. biomedcentral.com/1471-2407/10/615.

4. Combs SE, Adeberg S, Dittmar J-O, Welzel T, Rieken S, Habermehl D, et al. Skull base meningiomas: long-term results and patient self-reported outcome in 507 patients treated with fractionated stereotactic radiotherapy (FSRT) or intensity modulated radiotherapy (IMRT). Radiother Oncol. 2013; 106:186-91. https://doi.org/10.1016/j.radonc.2012.07.008.

5. Vernimmen FJ, Harris JK, Wilson JA, Melvill R, Smit BJ, Slabbert JP. Stereotactic proton beam therapy of skull base meningiomas. Int J Radiat Oncol. 2001;49:99-105. https://doi.org/10.1016/s0360-3016(00)01457-7.

6. Combs SE, Ganswindt U, Foote RL, Kondziolka D, Tonn J-C. State-of-the-art treatment alternatives for base of skull meningiomas: complementing and controversial indications for neurosurgery, stereotactic and robotic based radiosurgery or modern fractionated radiation techniques. Radiat Oncol. 2012;7:226. https://doi.org/10.1186/1748-717x-7-226.

7. Condra KS, Buatti JM, Mendenhall WM, Friedman WA, Marcus RB, Rhoton AL. Benign meningiomas: primary treatment selection affects survival. Int J Radiat Oncol. 1997;39:427-36. https://doi.org/10.1016/s0360-3016(97)00317-9.

8. Weber DC, Lomax AJ, Peter Rutz H, Stadelmann O, Egger E, Timmermann B, et al. Spot-scanning proton radiation therapy for recurrent, residual or untreated intracranial meningiomas. Radiother Oncol. 2004;71:251-8. https://doi.org/10.1016/j.radonc.2004.02.011.

9. Schulz-Ertner D, Tsujii H. Particle radiation therapy using proton and heavier ion beams. J Clin Oncol. 2007;25:953-64. https://doi.org/10.1200/jco.2006.09.7816.

10. Halasz LM, Bussière MR, Dennis ER, Niemierko A, Chapman PH, Loeffler JS, et al. Proton stereotactic Radiosurgery for the treatment of benign Meningiomas. Int J Radiat Oncol. 2011;81:1428-35. https://doi.org/10.1016/j.jirobp.2010.07.1991.

11. El Shafie RA, Habermehl D, Rieken S, Mairani A, Orschiedt L, Brons S, et al. In vitro evaluation of photon and raster-scanned carbon ion radiotherapy in combination with gemcitabine in pancreatic cancer cell lines. J Radiat Res. 2013;54(suppl 1):i113-9. http://jrr.oxfordjournals.org/ cgi/doi/10.1093/jrr/rrt052.

12. Kessel KA, Bohn C, Engelmann U, Oetzel D, Bougatf N, Bendl R, et al. Fiveyear experience with setup and implementation of an integrated database system for clinical documentation and research. Comput Methods Prog Biomed. 2014;114:206-17. https://doi.org/10.1016/j.cmpb.2014.02.002.

13. National Institutes of Health $\mathrm{NCl}$. Common Terminology Criteria for Adverse Events v4.0 (CTCAE). 2010. Disponible en: https://www.eortc.be/services/doc/ ctc/CTCAE_4.03_2010-06-14_QuickReference_5x7.pdf. Accessed 19 Dec 2016.

14. Schemper M, Smith TL. A note on quantifying follow-up in studies of failure time. Control Clin Trials. 1996;17:343-6. http://www.ncbi.nlm.nih.gov/ pubmed/8889347. Accessed 6 Jan 2018

15. Kessel KA, Fischer H, Oechnser M, Zimmer C, Meyer B, Combs SE. High-precision radiotherapy for meningiomas. Strahlenther Onkol. 2017;193:921-30. https://doi. org/10.1007/s00066-017-1156-3.

16. Kaul D, Budach V, Wurm R, Gruen A, Graaf L, Habbel P, et al. Linac-based stereotactic radiotherapy and radiosurgery in patients with meningioma. 2014;9:1-9. doi:https://doi.org/10.1186/1748-717X-9-78.

17. Kaul D, Budach V, Misch M, Wiener E, Exner S, Badakhshi H. Meningioma of the skull base: Long-term outcome after image-guided stereotactic radiotherapy Méningiomes de la base du crâne : résultats à long terme de la radiothérapie stéréotaxique guidée par l'image. Cancer / Radiother. 2014; 18:730-5. https://doi.org/10.1016/j.canrad.2014.07.159.

18. Minniti G, Clarke E, Cavallo L, Osti M, Esposito V, Cantore G, et al. Fractionated stereotactic conformal radiotherapy for large benign skull base meningiomas. Radiat Oncol. 2011;6:36. https://doi.org/10.1186/1748-717X-6-36.

19. Miralbell R, Cella L, Weber D, Lomax A. Optimizing radiotherapy of orbital and paraorbital tumors: intensity-modulated X-ray beams vs. intensitymodulated proton beams. Int J Radiat Oncol. 2000;47:1111-9. https://doi. org/10.1016/s0360-3016(00)00494-6.

20. Cozzi L, Fogliata A, Lomax A, Bolsi A. A treatment planning comparison of 3D conformal therapy, intensity modulated photon therapy and proton therapy for treatment of advanced head and neck tumours. Radiother Oncol. 2001;61:287-97. https://doi.org/10.1016/s0167-8140(01)00403-0. 
21. Lomax AJ, Cella L, Weber D, Kurtz JM, Miralbell R. Potential role of intensity-modulated photons and protons in the treatment of the breast and regional nodes. Int J Radiat Oncol. 2003;55:785-92. https:// doi.org/10.1016/s0360-3016(02)04210-4.

22. Hoskin PJ, Bhattacharya IS. Protons and more: state of the art in radiotherapy. Clin Med. 2014;14(Suppl 6):s61-5. http://eutils.ncbi.nlm.nih.gov/entrez/eutils/ elink.fcgi?dbfrom $=$ pubmed\&id $=25468922 \&$ retmode $=$ ref $\& \mathrm{cmd}=$ prlinks.

23. Bolsi A, Fogliata A, Cozzi L. Radiotherapy of small intracranial tumours with different advanced techniques using photon and proton beams: a treatment planning study. Radiother Oncol. 2003;68:1-14. https://doi.org/10. 1016/s0167-8140(03)00117-8.

24. Kosaki K, Ecker S, Habermehl D, Rieken S, Jäkel O, Herfarth K, et al. Comparison of intensity modulated radiotherapy (IMRT) with intensity modulated particle therapy (IMPT) using fixed beams or an ion gantry for the treatment of patients with skull base meningiomas. Radiat Oncol. 2012;7:44. http://www.rojournal.com/content/7/1/44

25. Noël G, Bollet MA, Calugaru V, Feuvret L, Haie-Meder C, Dhermain F, et al. Functional outcome of patients with benign meningioma treated by $3 \mathrm{D}$ conformal irradiation with a combination of photons and protons. Int J Radiat Oncol. 2005;62:1412-22. https://doi.org/10.1016/j.jirobp.2004.12.048.

26. Santacroce A, Walier M, Régis J, Liščák R, Motti E, Lindquist C, et al. Longterm tumor control of benign intracranial meningiomas after radiosurgery in a series of 4565 patients. Neurosurgery. 2012;70:32-9; discussion 39 https://doi.org/10.1227/NEU.0b013e31822d408a.

27. Murray FR, Snider JW, Bolsi A, Lomax AJ, Walser M, Kliebsch U, et al. Longterm clinical outcomes of pencil beam scanning proton therapy for benign and non-benign intracranial Meningiomas. Int J Radiat Oncol Biol Phys. 2017:99:1190-8. https://doi.org/10.1016/j.jirobp.2017.08.005.

28. DiBiase SJ, Kwok Y, Yovino S, Arena C, Naqvi S, Temple R, et al. Factors predicting local tumor control after gamma knife stereotactic radiosurgery for benign intracranial meningiomas. Int J Radiat Oncol. 2004;60:1515-9. https://doi.org/10.1016/j.jijobp.2004.05.073.

29. Sanford NN, Yeap BY, Larvie M, Daartz J, Munzenrider JE, Liebsch NJ, et al. Prospective, randomized study of radiation dose escalation with combined proton-photon therapy for benign Meningiomas. Int J Radiat Oncol Biol Phys. 2017;99:787-96. https://doi.org/10.1016/j.jirobp.2017.07.008.

30. Adeberg S, Hartmann C, Welzel T, Rieken S, Habermehl D, von Deimling A et al. Long-term outcome after radiotherapy in patients with atypical and malignant Meningiomas_clinical results in 85 patients treated in a single institution leading to optimized guidelines for early radiation therapy. Int J Radiat Oncol. 2012;83:859-64. https://doi.org/10.1016/j.jprobp.2011.08.010

31. McDonald MW, Plankenhorn DA, McMullen KP, Henderson MA, Dropcho EJ, Shah MV, et al. Proton therapy for atypical meningiomas. J Neuro-Oncol. 2015;123:123-8. https://doi.org/10.1007/s11060-015-1770-9.

32. Krämer M, Weyrather WK, Scholz M. The increased biological effectiveness of heavy charged particles: from radiobiology to treatment planning. Technol Cancer Res Treat. 2003;2:427-36. http://eutils.ncbi.nlm.nih.gov/entrez/eutils/ elink.fcgi?dbfrom=pubmed\&id $=14529307 \&$ retmode $=$ ref\&cmd=prlinks

33. Combs SE, Hartmann C, Nikoghosyan A, Jäkel O, Karger CP, Haberer T, et al. Carbon ion radiation therapy for high-risk meningiomas. Radiother Oncol. 2010;95:54-9. https://doi.org/10.1016/j.radonc.2009.12.029.

34. Phillips MH, Stelzer KJ, Griffin TW, Mayberg MR, Winn HR. Stereotactic radiosurgery: a review and comparison of methods. J Clin Oncol. 1994;12: 1085-99. https://doi.org/10.1200/JCO.1994.12.5.1085.

35. Phillips MH, Frankel KA, Lyman JT, Fabrikant Jl, Levy RP. Comparison of different radiation types and irradiation geometries in stereotactic radiosurgery. Int J Radiat Oncol. 1990;18:211-20. https://doi.org/10.1016/ 0360-3016(90)90286-s.

36. Emami B, Lyman J, Brown A, Cola L, Goitein M, Munzenrider JE, et al. Tolerance of normal tissue to therapeutic irradiation. Int J Radiat Oncol Biol Phys. 1991;21: 109-22.

37. Smith V, Verhey L, Serago CF. Comparison of radiosurgery treatment modalities based on complication and control probabilities. Int J Radiat Oncol. 1998;40:507-13. https://doi.org/10.1016/s0360-3016(97)00721-9.

\section{Submit your next manuscript to BioMed Central and we will help you at every step:}

- We accept pre-submission inquiries

- Our selector tool helps you to find the most relevant journal

- We provide round the clock customer support

- Convenient online submission

- Thorough peer review

- Inclusion in PubMed and all major indexing services

- Maximum visibility for your research

Submit your manuscript at www.biomedcentral.com/submit
C Biomed Central 